\title{
Slingshot homolog-1 expression is a poor prognostic factor of pT1 bladder urothelial carcinoma after transurethral resection
}

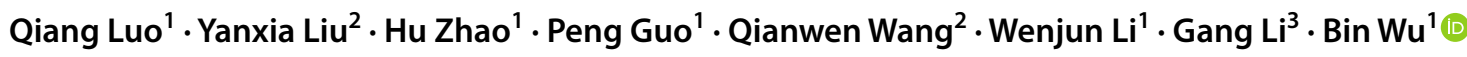

Received: 11 October 2019 / Accepted: 10 January 2020 / Published online: 21 January 2020

(c) The Author(s) 2020

\begin{abstract}
Objective Slingshot homolog-1 (SSH-1) shows an important role in the occurrence and development in various tumors. While, the expression and prognostic implications of SSH-1 in bladder urothelial carcinoma (UC) remain unclear and thus were addressed in this study.

Methods Immunohistochemistry (IHC) was performed on tissue microarrays composed of 624 bladder UC specimens after transurethral resection of bladder tumor (TUR-BT) to detect SSH-1 expression. The clinic-pathological features were compared between SSH-1(+) and SSH-1(-) subgroups. The Kaplan-Meier curve with log-rank test and univariate/multivariate Cox regression model with stepwise backward elimination methods were performed for survival analyses.

Results In this study, 359 (57.53\%) specimens were detected with SSH-1 expression. SSH-1 positivity was significantly associated with higher pathological grade $(p=0.020)$, lymphovascular invasion $(p=0.006)$, tumor recurrence $(p<0.001)$ and progression $(p<0.001)$ in bladder UC. Besides, SSH-1 positivity predicted a shorter overall survival (OS, $p=0.024)$, recurrence-free survival (RFS, $p<0.001$ ), progression-free survival (PFS, $p=0.002$ ) and cancer-specific survival (CSS, $p=0.047)$. Multivariate Cox proportional hazard analysis showed that tumor size $(p=0.007)$, lymphovascular invasion $(p=0.003)$, recurrence $(p<0.001)$, progression $(p<0.001)$ and SSH-1 expression $(p=0.015)$ were predictors of poor prognosis in bladder UC patients.

Conclusions SSH-1 expression was associated with undesirable clinic-pathological characteristics and poor post-operative prognosis in bladder UC patients. SSH-1 might play an important role in bladder UC and serve as a promising predictor of oncological outcomes in patients with bladder UC.
\end{abstract}

Keywords Slingshot homolog-1 $\cdot$ pT1 Bladder urothelial carcinoma $\cdot$ Transurethral resection $\cdot$ Prognostic factor

\section{Abbreviations}

SSH-1 Slingshot homolog-1

UC Urothelial carcinoma

IHC Immunohistochemistry

Qiang Luo and Yanxia Liu are co-first authors.

Bin Wu

13182796779@163.com

1 Department of Urology, Jiangyin People's Hospital, Affiliated Jiangyin Hospital of the Southeast University Medical College, No.163, Shoushan Rd, Jiangyin 214400, Jiangsu Province, China

2 Department of Pathology, Jiangyin People's Hospital, Affiliated Jiangyin Hospital of the Southeast University Medical College, Jiangyin 214400, Jiangsu Province, China

3 Department of Urology, Tianjin Institute of Urology, the Second Hospital of Tianjin Medical University, Tianjin 300211, China
TUR-BT Transurethral resection of bladder tumor

OS Overall survival

RFS Recurrence-free survival

PFS Progression-free survival

CSS Cancer-specific survival

BT Bladder tumor

NMIBC Non-muscle invasive bladder cancer

CIS Carcinoma in situ

UICC Union for International Cancer Control

BCG Bacillus Calmette-Guerin

EMT Epithelial-mesenchyma transformation

\section{Introduction}

Malignant bladder tumor (BT) is the ninth commonest cancer worldwide, accounting for approximately 390,000 cases and 150,000 deaths per year [1]. However, BT is the seventh 
most common cancer in men and ranks thirteen according to mortality from all malignant tumors. The incidence of BT in North America, Europe, and Western Asia is higher, but mortality rate is greater in developing countries [2]. Urothelial carcinoma (UC), also known as transitional cell carcinoma, is the most common pathological subtype, which accounts for more than $90 \%$ of malignant BT [3]. Transurethral resection of bladder tumor (TUR-BT) has been the standard treatment for initial, non-muscle invasive bladder cancer (NMIBC), including carcinoma in situ (CIS), stage Ta and T1 BT [4].

Slingshot homolog-1 (SSH-1) is a kind of protein phosphatase, which could dephosphorylate and activate cofilin specifically. Cofilin is a F-actin-severing protein. SSH-1 conglutinates and co-localizes with F-actin, then the cofilin-phosphatase activity of SSH-1 increases obviously by adhering to F-actin [5, 6]. SSH-1 likely be a critical factor for stimulus-induced actin remodeling and vascular smooth muscle cell migration. SSH-1 has been found overexpression in various cancers, including pancreatic cancer [7], gastric cancer [8], colorectal cancer [9], etc. The expression of SSH-1 usually predicts a poor prognosis and survival in patients [10]. A comprehensive research about the relationship of SSH-1 expression and bladder UC patients has not been reported. In present study, we investigated the expression of SSH-1 in bladder UC with immunohistochemistry (IHC) and clinic-pathological characteristics with aims to explore whether SSH-1 could serve as a prognostic indicator of stage pT1 bladder UC.

\section{Patients and methods}

\section{Patients}

This study was approved by the ethics committee of Jiangyin People's Hospital, and written informed consent was received from the subjects. A total of 624 bladder UC patients who underwent TUR-BT from Sep 2014 to Oct 2018 in our institution were enrolled in this study. No lymph node or distant metastasis was found through pre-operative examination in all patients. All resected specimens were reviewed by two experienced pathologists and diagnosed as stage pT1 bladder UC. The pathological stage was defined on the basis of the 2009 Union for International Cancer Control (UICC) TNM staging system, and tumor grading according to the 2004 WHO classification system for noninvasive urothelial system. All patients accepted intravesical chemotherapy regularly with epirubicin or gemcitabine and cystoscopy according to the EAU guidelines (2016) [11]. We did not use Bacillus Calmette-Guerin (BCG) because it has not been approved by China Food and Drug Administration before. The patients' clinic-pathological information was obtained from medical records (Table 1). Formalin-fixed paraffin-embedded tissue blocks of post-operative tissues were collected for IHC examinations. All patients were divided into two cohorts according to the expression of SSH1. To avoid the influence of tumor grade, we subsequently divided all cases into low-grade and high-grade subgroups with or without SSH-1 expression, respectively. Overall survival (OS), recurrence-free survival (RFS), progressionfree survival (PFS) and cancer-specific survival (CSS) were performed for the analysis of prognostic implications. The PFS duration was calculated from first operation to the date when the disease developed to a higher pathological stage, histological grade and/or to lymphatic or distant metastasis.

\section{IHC and scoring}

IHC staining was utilized to detect $\mathrm{SSH}-1$ expression in the post-operative bladder UC tissues. Rabbit anti-SSH-1

Table 1 Clinic-pathological characteristics of the SSH-1(+) and SSH-1(-) patients with bladder urothelial carcinoma

\begin{tabular}{|c|c|c|c|}
\hline Characteristics & $\begin{array}{l}\text { SSH-1(+) } \\
n=359(57.53 \%)\end{array}$ & $\begin{array}{l}\text { SSH-1(-) } \\
n=265(42.47 \%)\end{array}$ & $p$ value \\
\hline Mean age (years) & $63.7 \pm 15.5$ & $65.8 \pm 14.9$ & 0.462 \\
\hline \multicolumn{4}{|l|}{ Sex } \\
\hline Male & $281(78.27 \%)$ & $198(74.72 \%)$ & \\
\hline Female & $78(21.73 \%)$ & $67(25.28 \%)$ & 0.299 \\
\hline \multicolumn{4}{|l|}{ Tumor size $(\mathrm{cm})$} \\
\hline$<3 \mathrm{~cm}$ & $244(67.97 \%)$ & $186(70.19 \%)$ & \\
\hline$\geq 3 \mathrm{~cm}$ & $115(32.03 \%)$ & $79(29.81 \%)$ & 0.553 \\
\hline \multicolumn{4}{|l|}{ Pathological grade } \\
\hline Low & $156(43.45 \%)$ & $140(52.83 \%)$ & \\
\hline High & $203(56.55 \%)$ & $125(47.17 \%)$ & 0.020 \\
\hline \multicolumn{4}{|l|}{ Tumor multiplicity } \\
\hline Present & $143(39.83 \%)$ & $95(35.84 \%)$ & \\
\hline Absent & $216(60.17 \%)$ & $170(64.16 \%)$ & 0.311 \\
\hline \multicolumn{4}{|c|}{ Lymphovascular invasion } \\
\hline Present & $68(18.94 \%)$ & $29(10.94 \%)$ & \\
\hline Absent & $291(81.06 \%)$ & $236(89.06 \%)$ & 0.006 \\
\hline \multicolumn{4}{|c|}{ Squamous differentiation } \\
\hline Present & $74(20.61 \%)$ & $43(16.23 \%)$ & \\
\hline Absent & $285(79.39 \%)$ & $222(83.77 \%)$ & 0.165 \\
\hline \multicolumn{4}{|c|}{ Glandular differentiation } \\
\hline Present & $28(7.80 \%)$ & $16(6.04 \%)$ & \\
\hline Absent & $331(92.20 \%)$ & $249(93.96 \%)$ & 0.396 \\
\hline \multicolumn{4}{|l|}{ Recurrence } \\
\hline Present & $202(56.27 \%)$ & $101(38.11 \%)$ & \\
\hline Absent & $157(43.73 \%)$ & $164(61.89 \%)$ & $<0.001$ \\
\hline \multicolumn{4}{|l|}{ Progression } \\
\hline Present & $165(45.96 \%)$ & $76(28.68 \%)$ & \\
\hline Absent & $194(54.04 \%)$ & $189(71.32 \%)$ & $<0.001$ \\
\hline
\end{tabular}


antibody was purchased from Abcam. Co. (ab76943; Cambridge, UK) at a dilution of $1 / 1000$, according to the protocol. Yellow or brown staining indicated SSH-1 positivity. The SSH-1 staining scoring system is based on published research [8]. The intensity of SSH-1 in cancer cells was graded as $0-3$, and the number of SSH-1(+) cells was graded as $0-4$ according to the percentage of positive cells (0: 0-5\%; 1: 6-25\%; 2: 26-50\%; 3: 51-75\%; 4: 76-100\%). The sum of both grades defined as the final SSH-1 staining score (SSH-1(+): 4-7; SSH-1(-): 0-3) (Fig. 1).

\section{Statistical analysis}

All statistical analysis was calculated with SPSS 22.0 (Chicago, IL, USA). The student's $t$ test and Chi-square test were performed to compare the clinic-pathological features between SSH-1(+) and SSH-1(-) subgroups. The survival outcomes were evaluated by Kaplan-Meier curves and differences were calculated by the log-rank test. Univariate and multivariate Cox proportional hazards models were used to evaluate prognostic factors. All tests were two sided, and a $p$ value $<0.05$ was considered to be statistically significant.

\section{Results}

1. The association of SSH-1 expression in bladder UC specimens with clinic-pathological characteristics of included patients.

The clinic-pathological characteristics of all 624 patients are shown in Table 1. SSH-1(+) was discovered in $359(57.53 \%)$ cases of 624 bladder UC patients, SSH$1(-)$ was in $265(42.47 \%)$ cases. There was no statistical difference in mean age $(p=0.462)$, sex ratio $(p=0.299)$, tumor size $(p=0.553)$, tumor multiplicity $(p=0.311)$, squamous differentiation $(p=0.165)$ and glandular differentiation $(p=0.396)$ between SSH-1 $(+)$ and SSH$1(-)$ groups. While SSH-1 expression was significantly associated with higher pathological grade $(56.55 \%$ vs. $47.17 \%, p=0.020)$, lymphovascular invasion $(18.94 \%$ vs. $10.94 \%, p=0.006)$, and an increased occurrence of tumor recurrence $(56.27 \%$ vs. $38.11 \%, p<0.001)$ and progression $(45.96 \%$ vs. $28.68 \%, p<0.001)$. The lowgrade bladder UC in SSH-1(+) and SSH-1(-) patients were $156(43.45 \%)$ and $140(52.83 \%)$ cases, and high-
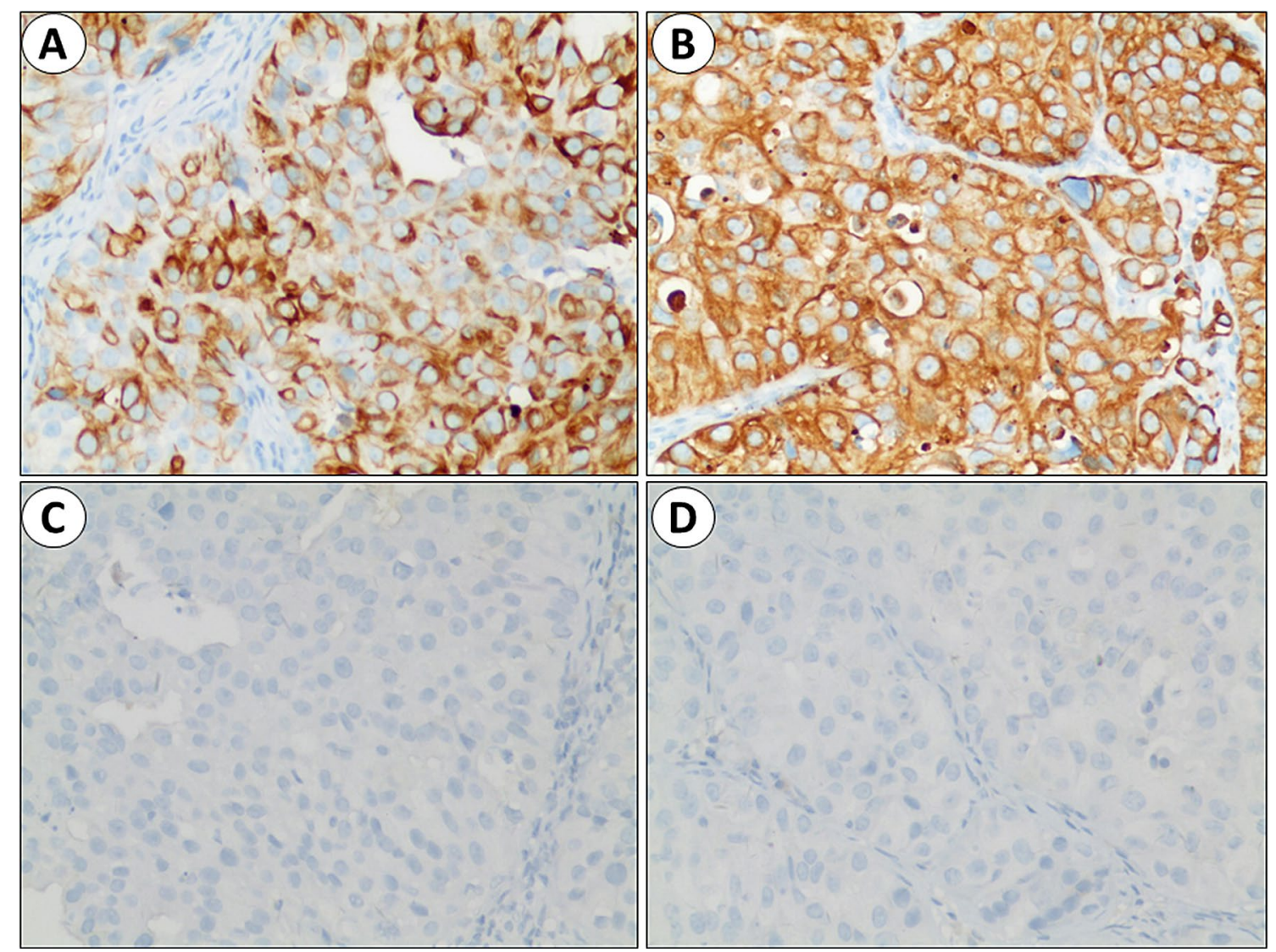

Fig. 1 Immunohistochemistry staining of SSH-1 expression in bladder UC specimens. a, b positive expression of SSH-1 in cancer tissues; $400 \times$ magnification. c, $\mathbf{d}$ negative expression of SSH-1 in cancer tissues; $400 \times$ magnification 
grade cases in two cohorts were 203 (56.55\%) and 125 (47.17\%), respectively.

2. The relationship between SSH-1 expression and patients' survival outcomes.

The average follow-up in present study was 36.64 months (range: 3 to 57). Kaplan-Meier curve and log-rank test demonstrated that patients with SSH-1(+) had a shorter OS $(p=0.024)$, RFS $(p<0.001)$, PFS $(p=0.002)$ and CSS $(p=0.047)$ than patients of SSH-1(-) group (Figs. 2a, 3a, $4 \mathrm{a}$ and $5 \mathrm{a})$. In subgroup of low-grade bladder UC, RFS $(p<0.001)$ and CSS $(p=0.029)$ were also shorter in SSH1(+) patients (Figs. 3b, 5b). However, no statistical difference was found in PFS $(p=0.126)$ and OS $(p=0.398)$ between the two subgroups (Figs. 2b, 4b). In subgroup of high-grade bladder UC, SSH-1 positivity was significantly associated with poor OS $(p=0.012)$, RFS $(p=0.002)$, PFS $(p<0.001)$ and CSS $(p=0.011)$ than SSH-1 negativity patients (Figs. 2c, 3c, $4 c$ and 5c).

The univariate analysis model indicated that tumor size $(\mathrm{HR}=1.566, p=0.015)$, pathological grade $(\mathrm{HR}=1.325$, $p=0.024)$, lymphovascular invasion $(\mathrm{HR}=1.838$,
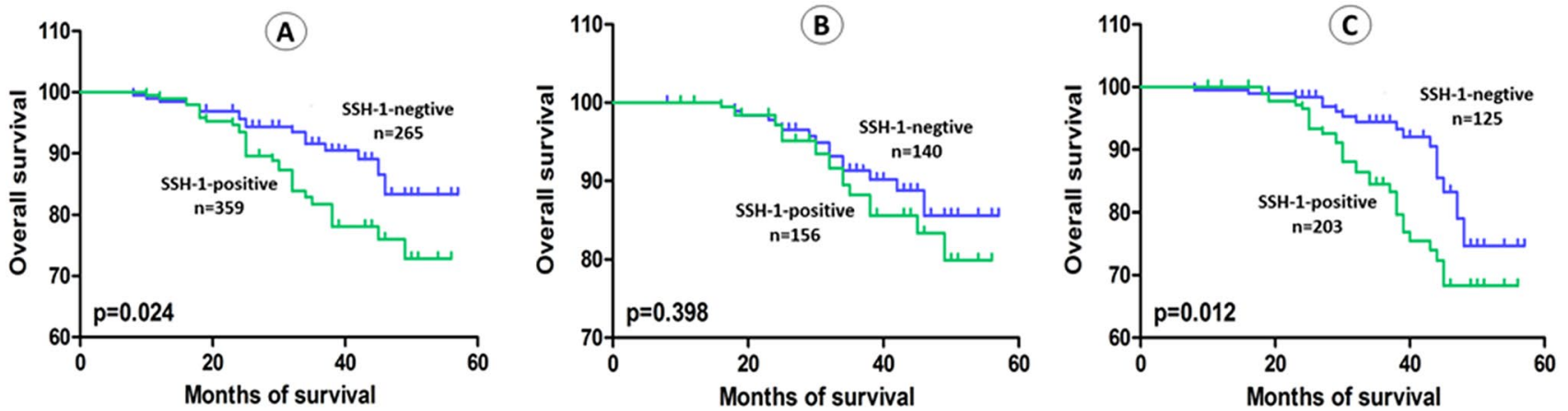

Fig. 2 Kaplan-Meier curve of the overall survival (OS) for all patients (a), low-grade (b) and high-grade subgroups (c)
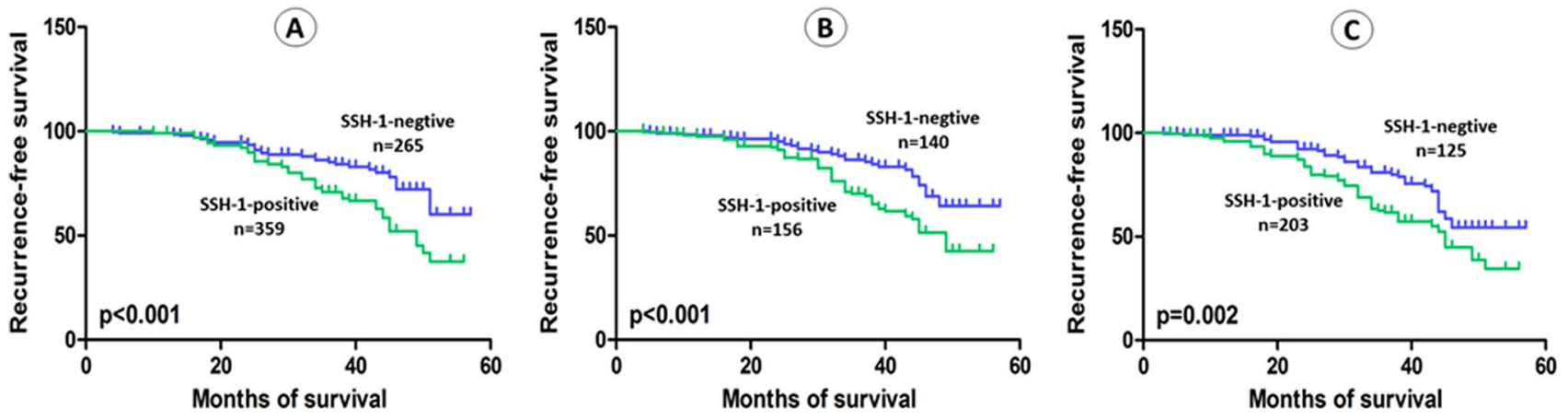

Fig. 3 Kaplan-Meier curve of the recurrence-free survival (RFS) for all patients (a), low-grade (b) and high-grade subgroups (c)
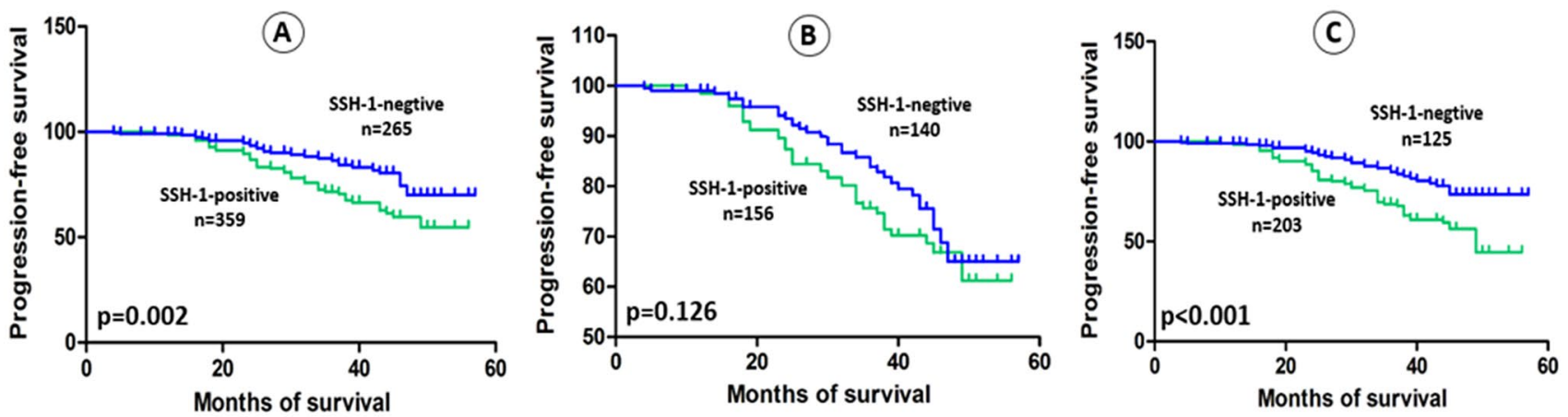

Fig. 4 Kaplan-Meier curve of the progression-free survival (PFS) for all patients (a), low-grade (b) and high-grade subgroups (c) 

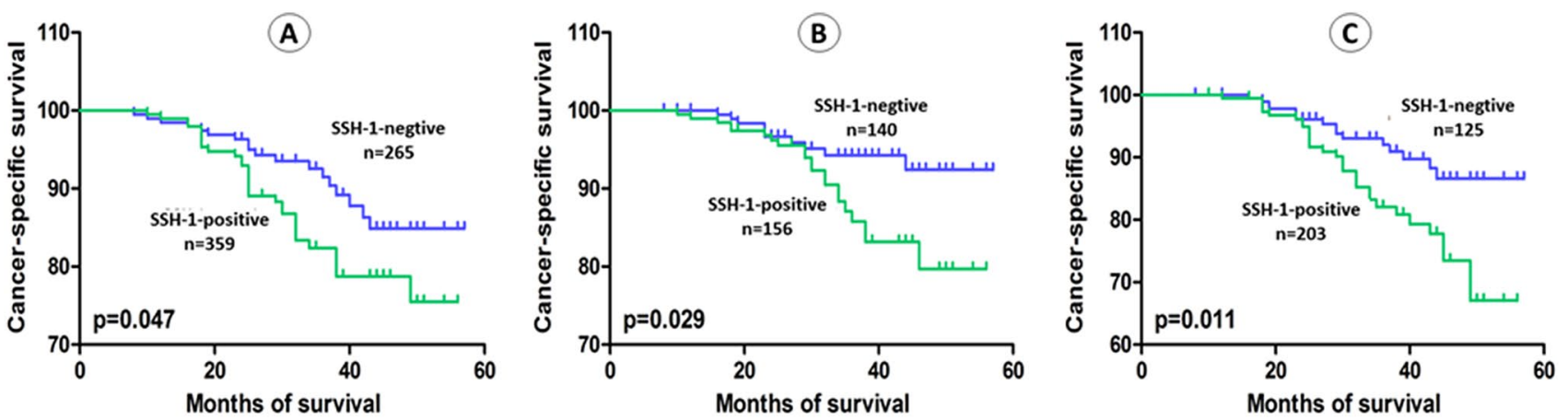

Fig. 5 Kaplan-Meier curve of the cancer-specific survival (CSS) for all patients (a), low-grade (b) and high-grade subgroups (c)

$p<0.001)$, squamous differentiation $(\mathrm{HR}=1.492$, $p=0.032)$, tumor recurrence $(\mathrm{HR}=3.451, p<0.001)$, progression $(\mathrm{HR}=2.947, p<0.001)$, and $\mathrm{SSH}-1$ positive expression $(\mathrm{HR}=1.693, p<0.001)$ showed prognostic implication for the predication of CCS in patients with bladder UC (Table 2). In the multivariate Cox regression model, tumor size $(\mathrm{HR}=1.429, p=0.007)$, lymphovascular invasion $(\mathrm{HR}=1.692, p=0.003)$, recurrence $(\mathrm{HR}=3.856$, $p<0.001)$, progression $(\mathrm{HR}=2.655, p<0.001)$ and SSH-1 positive expression $(\mathrm{HR}=1.558, p=0.015)$ were significant variables of CSS (Table 2). The results indicated that SSH-1 expression is a specific predictor of poor CSS for stage pT1 bladder UC patients.

\section{Discussions}

Bladder UC is the most common pathological subtype of malignant BT. Approximately $75 \%$ of bladder UC patients presented with non-muscle-invasion bladder cancer (NMIBC), including carcinoma in situ (CIS, 10\%), stage Ta (mucosa, 70\%) and T1 (submucosal invasive, 20\%). NMIBC is usually managed with complete TUR-BT and post-operative intravesical chemotherapy [12]. The surgery quality of initial TUR-BT directly influences the accurate diagnosis, pathological stage, adjuvant therapy, and survival prognosis of NMIBC. For NMIBC, some reports have showed that the 5-year recurrence rate after TUR-BT ranged from $50-70 \%$, and incomplete resection was considered the major reason [13]. The quality control indicators of TUR-BT include complete resection of visible tumors, second-look TUR-BT (or be called re-TUR-BT) for stage pT1 and high-grade BT, adjuvant intravesical chemotherapy or immunotherapy with BCG, presence of detrusor muscle in the excised tissues and no bladder perforation $[14,15]$. Although second-look TUR-BT has been recommended for patients with stage pT1 BT after initial operation for restaging and deciding the subsequent therapy. Some scholars queried the need for such additional surgical intervention, considering the patients and healthcare burdens, and the survival benefits of second-look TUR-BT is still controversial at present $[12,16]$. Hence, the majority of patients with stage pT1 bladder UC were not performed re-TUR-BT in our institution. To avoid the selection bias of included cases, patients who accepted re-TUR-BT after initial surgery were excluded in this study.
Table 2 Univariate and multivariate Cox regression analyses of cancer-specific survival of patients with bladder urothelial carcinoma

\begin{tabular}{|c|c|c|c|c|c|c|}
\hline \multirow[t]{2}{*}{ Variables } & \multicolumn{3}{|c|}{ Univariate analysis } & \multicolumn{3}{|c|}{ Multivariate analysis } \\
\hline & HR & $95 \% \mathrm{CI}$ & $p$ value & HR & $95 \% \mathrm{CI}$ & $p$ value \\
\hline Age (years) & 1.031 & $0.894-1.220$ & 0.463 & & & \\
\hline Sex & 1.108 & $0.912-1.365$ & 0.574 & & & \\
\hline Tumor size & 1.566 & $1.206-2.118$ & 0.015 & 1.429 & $1.283-1.804$ & 0.007 \\
\hline Pathological grade & 1.325 & $1.186-1.660$ & 0.024 & 1.213 & $0.944-1.589$ & 0.147 \\
\hline Tumor multiplicity & 1.291 & $0.847-1.693$ & 0.282 & & & \\
\hline Lymphovascular invasion & 1.838 & $1.337-2.506$ & $<0.001$ & 1.692 & $1.360-2.054$ & 0.003 \\
\hline Squamous differentiation & 1.492 & $1.255-1.906$ & 0.032 & 1.220 & $0.921-1.759$ & 0.188 \\
\hline Glandular differentiation & 1.190 & $0.751-1.543$ & 0.238 & & & \\
\hline Recurrence & 3.451 & $2.683-5.770$ & $<0.001$ & 3.856 & $3.007-5.402$ & $<0.001$ \\
\hline Progression & 2.947 & $2.380-4.136$ & $<0.001$ & 2.655 & $2.103-3.458$ & $<0.001$ \\
\hline SSH-1 expression & 1.693 & $1.235-2.106$ & $<0.001$ & 1.558 & $1.192-2.043$ & 0.015 \\
\hline
\end{tabular}


The SSH family protein phosphatases contain a highly conserved non-catalytic region (also be called "SSH-N domain"), which closes to the N-terminus of phosphatase catalytic domain $[17,18]$. The N-terminal half of SSH-1 (amino acids 1-461) has the ability of cofilin-phosphatase and F-actin-mediated activation. This reminded us that SSH-1 might play an important role in determining the substrate specificity of SSH-1 cytokine and in mediating F-actin-mediated activation of the cofilin-phosphatase [19]. Some evidences have confirmed that the activation of cofilin was critical for membrane protrusion formation, directional cell migration, and cancer cell invasion. Cofilin phosphoregulation by SSH-1 is a pivotal factor of actin cytoskeletal remodeling and involving in cancerous cells migration and metastasis [20]. Aggelou et al. [9] discovered that LIM kinases 1, LIM kinases 2 and SSH-1 were regulators of actin dynamics cofilin and contributed to colorectal cancer progression and chemoresistance to 5-fluorouracil. These cytokines were also associated with epithelial-mesenchyma transformation (EMT) markers, such as downregulation of E-cadherin and positive expression of ZEB. Wang et al. [7] found that the expression of SSH-1 was to be upregulated in pancreatic cancer cell lines with high metastatic potential, and loss of SSH-1 was correlated with an increase in the phosphorylation of cofilin- 1 and the inhibition of cancerous cell migration (but not proliferation). Moreover, SSH-1 was also significantly associated with lymph node metastasis in gastric cancer patients, and was an independent predictor of poor clinical outcomes in Maimaiti's research [8]. While, little is known regarding the SSH-1 expression in human stage pT1 bladder UC patients.

In present study, we focused on the prognostic significance of SSH-1 expression in stage pT1 bladder UC because patients with this disease after TUR-BT generally suffer from tumor recurrence and progression to invasive cancers $[11,15]$. A total of 624 bladder UC tissues were brought into this study, and 359 (57.53\%) cases showed SSH-1 positivity. The presence of SSH-1 in bladder UC specimens was significantly associated with unfavorable clinic-pathological characteristics, including higher pathological grade, lymphovascular invasion, and incidence of tumor recurrence and progression. Through follow-up and survival analysis, we identified that bladder UC patients with SSH-1 expression had a significantly poor OS, RFS, PFS, and CSS. We subsequently divided these patients into low-grade and highgrade subgroups on the basis of pathological examination. In the subgroup analysis, $\mathrm{SSH}-1$ positivity patients were still statistically associated with poorer oncological outcomes than SSH-1 negativity subgroup. Only in the PFS and OS of low-grade bladder UC patients, no significant difference was discovered between the two subgroups. Several predictors or prognostic markers for tumor recurrence, progression or cancer-specific death of bladder UC patients had been reported in previous studies, such as sex, age, tumor size, tumor multiplicity, pathological grade, CIS, T1 substaging, histologic features and $\mathrm{Ki}-67$ proliferation index [21-23]. Additionally, some scholars pointed that the presence of squamous and/or glandular differentiation was correlated with a higher recurrence rate and poor survival in NMIBC patients, such cellular heterogeneity could also be an independent prognostic predictor of bladder cancer [24, 25]. Nevertheless, Kim et al. [26] revealed the survival outcomes of bladder UC patients with squamous and/or glandular differentiation were similar to patients with pure bladder UC, given comparable demographic, clinic-pathologic and management features. Bladder UC with differentiation just presented with higher pathologic stage. Similar results had been reported in other literatures as well $[27,28]$. In this study, the multivariate Cox regression analysis showed that tumor size, lymphovascular invasion, recurrence, progression, and SSH-1 positivity were prognostic indicators influencing the CSS of stage pT1 bladder UC patients. Other variables, including pathological grade, tumor multiplicity, squamous and glandular differentiation had no significant impacts on the CSS of patients.

There are three limitations in our study. First, this is a retrospective research and inherent biases were unavoidable in patients' selection and treatment. Second, we did not recruit a large number of patients from multiple medical centers, and some patients in this study were not followed up for a long time. Third, we did not perform the molecular experiments and explore the mechanisms of this phenotype.

\section{Conclusions}

This study first concentrated on the oncologic outcomes of SSH-1 expression in stage pT1 bladder UC patients. We clarified the clinic-pathological significance of SSH-1 expression in such patients and discovered that $\mathrm{SSH}-1$ was significantly associated with an increased risk of tumor recurrence and progression. SSH-1 might be an independent predictor and promising biomarker for the treatment and/ or progression of pT1 bladder UC.

Author contribution QL, YXL, and BW conceived and designed the study. QL, PG, and HZ contributed to the writing of the manuscript. WJL, QWW, and GL collected and analyzed the data. YXL and QWW performed pathological examination. All authors read and approved the final manuscript.

Funding This work was supported by National Natural Science Foundation of China (Grants 81772756).

Data availability The data and materials of this study are available from the corresponding author upon reasonable request. 


\section{Compliance with ethical standards}

Conflict of interest The authors declare they have no conflict of interest.

Ethical approval This study has been approved by the ethics committee of Jiangyin People's Hospital and has been performed according to the ethical standards laid down in the 1964 Declaration of Helsinki.

Open Access This article is licensed under a Creative Commons Attribution 4.0 International License, which permits use, sharing, adaptation, distribution and reproduction in any medium or format, as long as you give appropriate credit to the original author(s) and the source, provide a link to the Creative Commons licence, and indicate if changes were made. The images or other third party material in this article are included in the article's Creative Commons licence, unless indicated otherwise in a credit line to the material. If material is not included in the article's Creative Commons licence and your intended use is not permitted by statutory regulation or exceeds the permitted use, you will need to obtain permission directly from the copyright holder. To view a copy of this licence, visit http://creativecommons.org/licenses/by/4.0/.

\section{References}

1. Antoni S, Ferlay J, Soerjomataram I, Znaor A, Jemal A, Bray F (2017) Bladder cancer incidence and mortality: a global overview and recent trends. Eur Urol 71(1):96-108

2. Cumberbatch MGK, Jubber I, Black PC, Esperto F, Figueroa JD, Kamat AM et al (2018) Epidemiology of bladder cancer: a systematic review and contemporary update of risk factors in 2018. Eur Urol 74(6):784-795

3. Mukherjee P, George AJP, Yadav BK, Jeyaseelan L, Kumar RM, Mukha RP et al (2019) The impact of narrow band imaging in the detection and resection of bladder tumor in transitional cell carcinoma of the bladder: a prospective, blinded sequential intervention randomized controlled Trial. Urology 128:55-61

4. Richards KA, Smith ND, Steinberg GD (2014) The importance of transurethral resection of bladder tumor in the management of nonmuscle invasive bladder cancer: a systematic review of novel technologies. J Urol 191(6):1655-1664

5. Torres RA, Drake DA, Solodushko V, Jadhav R, Smith E, Rocic $P$ et al (2011) Slingshot isoform-specific regulation of cofilinmediated vascular smooth muscle cell migration and neointima formation. Arterioscl Throm Vasc 31(11):2424-2431

6. Takahashi K, Okabe H, Kanno SI, Nagai T, Mizuno K (2017) A pleckstrin homology-like domain is critical for F-actin binding and cofilin-phosphatase activity of Slingshot-1. Biochem Biophys Res 482(4):686-692

7. Wang Y, Kuramitsu Y, Kitagawa T, Baron B, Yoshino S, Maehara $S$ et al (2015) Cofilin-phosphatase slingshot-1L (SSH1L) is overexpressed in pancreatic cancer (PC) and contributes to tumor cell migration. Cancer Lett 360(2):171-176

8. Maimaiti Y, Maimaitiming M, Li Y, Aibibula S, Ainiwaer A, Aili A et al (2018) SSH1 expression is associated with gastric cancer progression and predicts a poor prognosis. BMC Gastroenterol 18(1):12

9. Aggelou H, Chadla P, Nikou S, Karteri S, Maroulis I, Kalofonos $\mathrm{HP}$ et al (2018) LIMK/cofilin pathway and Slingshot are implicated in human colorectal cancer progression and chemoresistance. Virchows Arch 472(5):727-737

10. Ramirez-Munoz R, Castro-Sanchez P, Roda-Navarro P (2016) Ultrasensitivity in the cofilin signaling module: a mechanism for tuning T cell responses. Front Immunol 7:59
11. Babjuk M, Bohle A, Burger M, Capoun O, Cohen D, Comperat EM et al (2017) EAU guidelines on non-muscle-invasive urothelial carcinoma of the bladder: update 2016. Eur Urol 71(3):447-461

12. Cumberbatch MGK, Foerster B, Catto JWF, Kamat AM, Kassouf W, Jubber I et al (2018) Repeat transurethral resection in nonmuscle-invasive bladder cancer: a systematic review. Eur Urol 73(6):925-933

13. Akand M, Muilwijk T, Raskin Y, De Vrieze M, Joniau S, Van Der Aa F (2019) Quality control indicators for transurethral resection of non-muscle-invasive bladder cancer. Clin Genitourin Cancer 17(4):e784-e792

14. Kassouf W, Aprikian A, Black P, Kulkarni G, Izawa J, Eapen L et al (2016) Recommendations for the improvement of bladder cancer quality of care in Canada: a consensus document reviewed and endorsed by Bladder Cancer Canada (BCC), Canadian Urologic Oncology Group (CUOG), and Canadian Urological Association (CUA), December 2015. Can Urol Assoc 10(1-2):E46-80

15. Power NE, Izawa J (2016) Comparison of guidelines on non-muscle invasive bladder cancer (EAU, CUA, AUA, NCCN, NICE). Bladder Cancer 2(1):27-36

16. Calo B, Chirico M, Fortunato F, Sanguedolce F, Carvalho-Dias E, Autorino R et al (2019) Is Repeat transurethral resection always needed in high-grade t1 bladder cancer? Front Oncol 9:465

17. Ohta Y, Kousaka K, Nagata-Ohashi K, Ohashi K, Muramoto A, Shima Y et al (2003) Differential activities, subcellular distribution and tissue expression patterns of three members of Slingshot family phosphatases that dephosphorylate cofilin. Genes Cells 8(10):811-824

18. Mizuno K (2013) Signaling mechanisms and functional roles of cofilin phosphorylation and dephosphorylation. Cell Signal 25(2):457-469

19. Kurita S, Watanabe Y, Gunji E, Ohashi K, Mizuno K (2008) Molecular dissection of the mechanisms of substrate recognition and F-actin-mediated activation of cofilin-phosphatase Slingshot-1. J Biol Chem 283(47):32542-32552

20. Shishkin S, Eremina L, Pashintseva N, Kovalev L, Kovaleva M (2016) Cofilin-1 and other ADF/cofilin superfamily members in human malignant cells. Int J Mol Sci 18(1):10

21. Palou J, Sylvester RJ, Faba OR, Parada R, Pena JA, Algaba F et al (2012) Female gender and carcinoma in situ in the prostatic urethra are prognostic factors for recurrence, progression, and disease-specific mortality in T1G3 bladder cancer patients treated with Bacillus Calmette-Guerin. Eur Urol 62(1):118-125

22. Den V, Cerruto MA, D'Elia C, Brunelli M, Otte O, Minja A et al (2014) Prognostic role of substaging in T1G3 transitional cell carcinoma of the urinary bladder. Mol Clin Oncol 2(4):575-580

23. Gontero P, Sylvester R, Pisano F, Joniau S, Vander Eeckt K, Serretta $\mathrm{V}$ et al (2015) Prognostic factors and risk groups in T1G3 non-muscle-invasive bladder cancer patients initially treated with Bacillus Calmette-Guerin: results of a retrospective multicenter study of 2451 patients. Eur Urol 67(1):74-82

24. Tang Q, Xiong G, Li X, Fang D, Xi C, Zhang L et al (2016) The prognostic impact of squamous and glandular differentiation for upper tract urothelial carcinoma patients after radical nephroureterectomy. World J Urol 34(6):871-877

25. Xu H, Xie L, Liu X, Zhang Y, Shen Z, Chen T et al (2017) Impact of squamous and/or glandular differentiation on recurrence and progression following transurethral resection for non-muscle invasive urothelial carcinoma of bladder. Oncol Lett 14(3):3522-3528

26. Kim SP, Frank I, Cheville JC, Thompson RH, Weight CJ, Thapa P et al (2012) The impact of squamous and glandular differentiation on survival after radical cystectomy for urothelial carcinoma. J Urol 188(2):405-409

27. Xylinas E, Rink M, Robinson BD, Lotan Y, Babjuk M, Brisuda A et al (2013) Impact of histological variants on oncological 
outcomes of patients with urothelial carcinoma of the bladder treated with radical cystectomy. Eur J Cancer 49(8):1889-1897

28. Mitra AP, Bartsch CC, Bartsch G Jr, Miranda G, Skinner EC, Daneshmand S (2014) Does presence of squamous and glandular differentiation in urothelial carcinoma of the bladder at cystectomy portend poor prognosis? An intensive case-control analysis. Urol Oncol 32(2):117-127
Publisher's Note Springer Nature remains neutral with regard to jurisdictional claims in published maps and institutional affiliations. 